Filomat 30:14 (2016), 3855-3866

DOI 10.2298/FIL1614855S

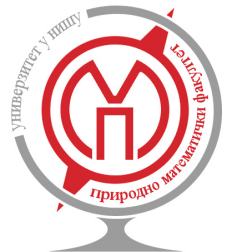

Published by Faculty of Sciences and Mathematics, University of Niš, Serbia

Availableat: http://www.pmf .ni.ac.rs/filomat

\title{
On Removing Blur in Images Using Least Squares Solutions
}

\author{
Predrag Stanimirovića ${ }^{a}$ Igor Stojanović ${ }^{b}$, Dimitrios Pappas $^{c}$, Spiros Chountasis ${ }^{\mathrm{d}}$, Zoran Zdravev ${ }^{\mathrm{b}}$ \\ ${ }^{a}$ University of Niš, Faculty of Sciences and Mathematics, Višegradska 33, 18000 Niš, Serbia \\ ${ }^{b}$ Goce Delčev University, Faculty of Computer Science, Goce Delčev 89, 2000 Štip, Macedonia \\ ${ }^{c}$ Department of Statistics, Athens University of Economics and Business, 76 Patission Str, 10434, Athens, Greece \\ ${ }^{d}$ Independent Power Transmission Operator, Asklipiou 22, GR-14568, Krioneri, Athens , Greece
}

\begin{abstract}
The further investigation of the image restoration method introduced in $[19,20]$ is presented in this paper. Continuing investigations in that area, two additional applications of the method are investigated. More precisely, we consider the possibility to replace the available matrix in the method by the restoration obtained applying the Tikhonov regularization method or the Truncated Singular Value decomposition method. Additionally, statistical analysis of numerical results generated by applying the proposed improvement of image restoration methods is presented. Previously performed numerical experiments as well as new numerical results and the statistical analysis confirm that the least squares approach can be used as a useful tool for improving restored images obtained by other image restoration methods.
\end{abstract}

\section{Introduction}

The motion blur is an outcome which appears in photographs of scenes where objects are moving. It is most obvious when the exposure is long, or if objects in the scene are moving rapidly. The field of image restoration refers to the estimation of the undamaged image from the blurred one.

Image restoration has caused a tremendous growth in interest over the last two decades. There are a lot of comprehensive overview articles, journal papers, and textbooks on the subject of image restoration and identification [1-3, 8, 9, 11-14]. The image restoration methods have been applied in different areas, such as the medical imaging and diagnosis, the satellite and astronomical imaging, the military surveillance, and remote sensing.

The image deblurring approach based on the usage of the Moore-Penrose inverse and least squares solutions of specific Toeplitz matrices, that appears in the corresponding mathematical model, is followed in the present paper. This approach was firstly used in [4,5]. A simplified implementation of the partitioning method on this class of Toeplitz matrices is described in [21]. The method based on the straightforward construction of the Moore-Penrose inverse of the blurring matrix is presented in [15].

2010 Mathematics Subject Classification. Primary 15A09; Secondary 68U10, 94A08

Keywords. Image restoration, Matrix equation, Least squares solution, Moore-Penrose inverse, Tikhonov regularization, Truncated Singular Value Decomposition

Received: 31 October 2014; Accepted: 07 January 2015

Communicated by Dragana Cvetkovic-Ilić

The first and second author gratefully acknowledge support from the Research Project 174013 of the Serbian Ministry of Science. The first, second and fifth author gratefully acknowledge support from the Project "Applying direct methods for digital image restoring" of the Goce Delčev University.

Email addresses: pecko@pmf.ni.ac.rs (Predrag Stanimirović), igor.stojanovik@ugd.edu.mk (Igor Stojanović), pappdimitris@gmail.com (Dimitrios Pappas), schountasis@admie.gr (Spiros Chountasis), zoran.zdravev@ugd.edu.mk (Zoran Zdravev) 
The main purpose of this article is further investigation of the algorithm, introduced in $[19,20]$, that allows us to remove a linear motion blur from images. The algorithm is based on the least squares solution of a matrix equation which represents the mathematical model of the linear motion blur. The least squares solution includes the Moore-Penrose inverse of the matrix which causes the linear motion blur as well as an arbitrary matrix $Y$. Satisfactory results are obtained when the matrix $Y$ is suitably defined. In this work we will show that it is possible to use the Tikhonov (TIK) and Truncated Singular Value Decomposition (TSVD) image restoration methods as two possible feasible approaches to generate the matrix $Y$.

The least squares method is a frequently used technique for solving various problems. An application of least-squares, regularization and fourth-order partial differential equations to restore degraded image is proposed in [22]. In addition, the least-squares method is used for the construction of an approximate solution of a linear ill-posed boundary-value problem [6], as well as in computation of the weighted Moore-Penrose inverse [17].

This paper is organized as follows. The motivation and description of the necessary methods are presented in the second section. The Truncated Singular Value Decomposition (TSVD) and the Tikhonov method (TIK) are restated from [9] in the third section. Furthermore, the experimental and numerical results derived by applying the operator $E$ on the restorations $Y=T S V D$ and $Y=T I K$ are described in the same section. Finally, the statistical description of results in $[19,20]$ and also in Section 3 is presented in Section 4.

\section{Mathematical Models}

Our improvement of image restoration methods assumes that the characteristics of the degrading system are known a priori. We start from the mathematical model (2.1) from [19], in which the linear motion is a local phenomenon and no additional noise is included. This model relates an arbitrary $i$ th row $g_{i}=\left[g_{i, 1}, \ldots, g_{i, m}\right]$ of the blurred image $G \in \mathbb{R}^{p \times m}$ with corresponding $i$ th row $f_{i}=\left[f_{i, 1}, \ldots, f_{i, m}\right]$ of the original image $F_{0}$, by the matrix equation

$$
g_{i}^{T}=\left[H_{-1}\left|H_{0}\right| H_{1}\right]\left[\frac{f_{i,-1}}{\frac{f_{i}^{T}}{f_{i, 1} \cdot}}\right] .
$$

The vectors $f_{i,-1}$ and $f_{i,-1}$ are defined by

$$
f_{i,-1}=\left[\begin{array}{c}
w_{i, 1} \\
\vdots \\
w_{i, u}
\end{array}\right], f_{i, 1}=\left[\begin{array}{c}
v_{i, 1} \\
\vdots \\
v_{i, u}
\end{array}\right],
$$

where $w_{i, 1}, \ldots, w_{i, u}$ and $v_{i, 1}, \ldots, v_{i, u}$ are boundary pixels. The boundary pixels left of the horizontal line are added above the initial vector $f_{i}^{T} \in \mathbb{R}^{m}$. The $u$ boundary pixels right of the horizontal line are added below the vector $f_{i}[9]$. Further, the matrices

$$
H_{-1} \in \mathbb{R}^{m \times u}, H_{1} \in \mathbb{R}^{m \times u}, H_{0} \in \mathbb{R}^{m \times m}
$$

are determined implicitly in the block matrix

$$
H=\left[H_{-1}\left|H_{0}\right| H_{1}\right],
$$

which is defined as 


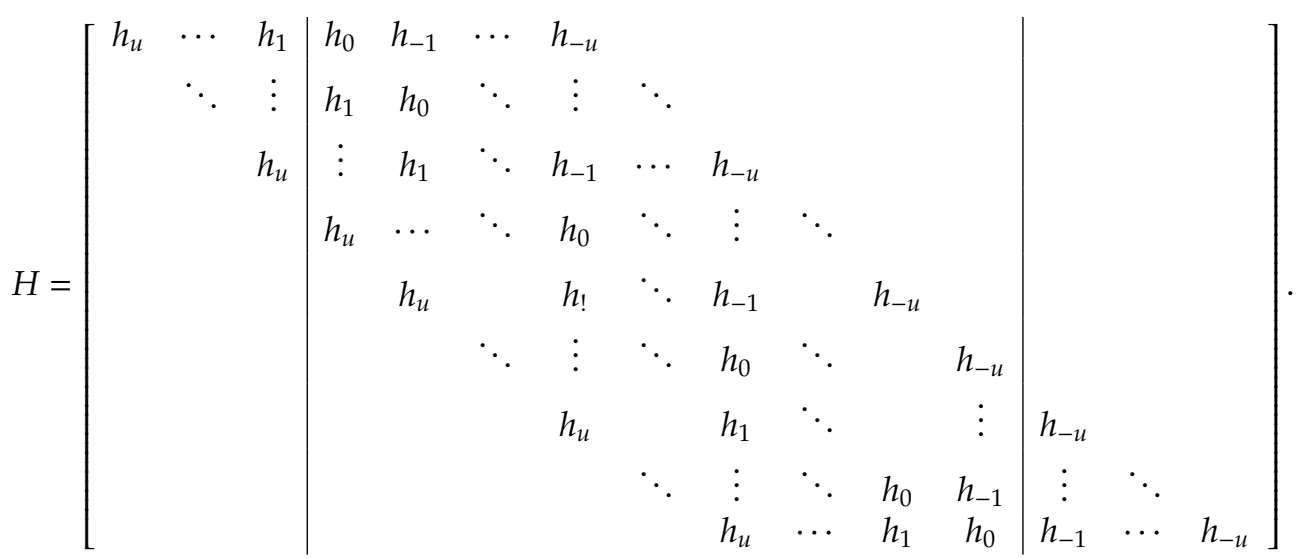

The elements $h_{i}$ are real numbers and $l-1=2 u$, where the integer $l$ indicates the length of linear motion blur in pixels. The mathematical model (2.1) is reused mainly from [7,9]. The matrix $H$ is $m \times s$ real matrix satisfying $s=m+l-1=m+2 u, m \gg l$.

The objective is to estimate the original image $F_{0} \in \mathbb{R}^{p \times m}$ row per row (contained in the vector $f_{i}^{T}$ ), by exploiting the given row of a blurred image (contained in the vector $g_{i}^{T}$ ) and a priori knowledge of the degradation phenomenon $H$. We will denote by $F_{-1}$ (resp. by $F_{1}$ ) the matrix whose columns are $f_{i,-1}$ (resp. $\left.f_{i, 1}\right)$. Then it is possible to consider the block matrix

$$
F=\left[F_{-1}\left|F_{0}^{T}\right| F_{1}\right] \in \mathbb{R}^{p \times s},
$$

in which the blocks satisfy $F_{-1} \in \mathbb{R}^{p \times u}, F_{1} \in \mathbb{R}^{p \times u}, F_{0} \in \mathbb{R}^{p \times m}$.

Then the equation (2.1) can be written in condensed form as follows:

$$
\begin{aligned}
G & =\left(\left[H_{-1}\left|H_{0}\right| H_{1}\right]\left[\begin{array}{c}
F_{-1} \\
F_{0}^{T} \\
F_{1}
\end{array}\right]\right)^{T}=\left[F_{-1}^{T}\left|F_{0}\right| F_{1}^{T}\right]\left[\begin{array}{c}
H_{-1}^{T} \\
H_{0}^{T} \\
H_{1}^{T}
\end{array}\right] \\
& =F H^{T}, G \in \mathbb{R}^{p \times m}, H \in \mathbb{R}^{m \times s}, F \in \mathbb{R}^{p \times s}, s=m+n-1 .
\end{aligned}
$$

We use the zero (Dirichlet), periodic and reflective boundary conditions (BCs) from [18]. Details can also be found also in [19].

A new approach in the restoration of a blurred image, which exploits a set of least squares solutions of the matrix equation (2.2), is proposed in the papers $[19,20]$. The least squares solutions are generated using the Moore-Penrose inverse. The Moore-Penrose inverse of a matrix $A \in \mathbb{C}^{m \times n}$ is the unique matrix, denoted by $A^{\dagger}$, satisfying the following four matrix equations:

$$
\begin{aligned}
& \text { (1) } A X A=A \\
& \text { (2) } X A X=X \\
& \text { (3) }(A X)^{*}=A X \\
& \text { (4) }(X A)^{*}=X A \text {. }
\end{aligned}
$$

A matrix $X$ is called an $\{i, j, k\}$-inverse of $A$ (with $i, j, k \in\{1,2,3,4\}$ ) if $X$ satisfies the $i$ th, $j$ th and $k$ th Penrose equations.

The general solution of the the matrix equation (2.2) is given by

$$
E(Y)=\widetilde{F}=G\left(H^{T}\right)^{\dagger}+Y\left(I-H^{\dagger} H\right),
$$

where the matrix $Y \in \mathbb{R}^{p \times s}$ is a disposable matrix which can be randomly chosen. Our original intention was to use values for $Y$ as close as possible to the original image $F$ in order to produce better restoration of the blurred image $G$. The results generated in the case $Y=O$ produces the Moore-Penrose solution of (2.2):

$$
\hat{F}=G\left(H^{T}\right)^{\dagger} .
$$


This particular choice corresponds to the best approximate solution (i.e. the Moore-Penrose solution) of the matrix equation (2.2), and it is investigated in [4,5]. Furthermore, we have observed in $[19,20]$ that the operator $E(Y)$ frequently gives a better improvement of blurred image with respect to restoration contained in $Y$. Therefore, the method proposed in $[19,20]$ is an improvement of image restoration methods. The main purpose of this paper is to analyze statistically the improvement that which is obtained by using the operator $E(Y)$ with respect to the restoration $Y$.

There is no practical reason to consider the operator $E(Y)$ as independent image restoration method. The full meaning of the operator $E(Y)$ is reflected in a symbiosis with the image restoration method $Y$. Therefore, it has been tested against well known image restoration methods. If the image restoration method is denoted by $Y$, then the improvement is denoted by $E(Y)$. In what follows we compare the values of Improvement in Signal-To-Noise Ratio (denoted by $I S N R(Y)$ ) as well as the values of Peak Signal-To-Noise Ratio (denoted by $P S N R(Y)$ ). More precisely, we compare the values $I S N R(Y)$ (resp. PSNR $(Y)$ ) against corresponding values of $\operatorname{ISNR}(E(Y))$ (rep. PSNR(E(Y))). The comparison of ISNR and PSNR values corresponding to $E(Y)$ with other image restoration methods is not of interest; it is only meaningful to compare values corresponding to restorations $Y$ with corresponding restorations $E(Y)$. In general, any image restoration method may be used to produce a restoration $Y$ of a blurred image. Continuing the investigation from [19,20], we propose restorations produced by the Truncated Singular Value Decomposition and the Tikhonov reconstruction of the blurred image as two possible generators of the matrix $Y$. For the sake of completeness, these methods are briefly restated in the next section.

\section{Improving Tikhonov and TSVD Methods}

The Truncated Singular Value Decomposition (TSVD shortly) and the Tikhonov (shortly denoted by TIK) image restoration methods from [9] are restated in this section. Further, the experimental and numerical results derived applying restorations $E(Y=T S V D)$ and $E(Y=T I K)$ are considered.

\subsection{Overview of Tikhonov and TSVD image restoration methods}

The Singular Value Decomposition (SVD) is a matrix computation tool for analyzing the system of linear equations $A x=b$. The vectors $x$ and $b$ are long vectors obtained by stacking the columns from the images $X$ and $B$. The matrix $X \in \mathbb{R}^{m \times n}$ is the desired sharp image, the matrix $B \in \mathbb{R}^{m \times n}$ is the recorded blurred image and the blurring matrix $A \in \mathbb{R}^{N \times N}$ has both dimensions $N=m * n$. The following SVD of the matrix $A$ is used (see, for example [9]):

$$
A=U \Sigma V^{T},
$$

where $U$ and $V$ are orthogonal matrices which satisfy $U^{T} U=I_{N}$ and $V^{T} V=I_{N}$. The matrix $\Sigma$ is a diagonal matrix whose entries are the singular values $\sigma_{1} \geq \sigma_{2} \geq \cdots \geq \sigma_{N} \geq 0$ of $A$.

Both TSVD and TIK methods belong to the family of the spectral filtering methods, because they give us control on the spectral content of the deblurred image with the filter factors $\phi_{i}$ [9]. In accordance with this approach, approximate solution of the linear system $A x=b$ is equal to

$$
x_{\text {filt }}=\sum_{i=1}^{N} \phi_{i} \frac{u_{i}^{T} b}{\sigma_{i}} v_{i},
$$

where the columns $u_{i}$ of $U$ are called the left singular vectors and the columns $v_{i}$ of $V$ represent the right singular vectors. From $U^{T} U=I_{N}$ (resp. $V^{T} V=I_{N}$ ) it follows that $u_{i}^{T} u_{j}=0, i \neq j\left(\right.$ resp. $v_{i}^{T} v_{j}=0, i \neq j$ ). Different spectral filtering algorithms are obtained by means of different selections of the filter factors. The most popular between them are TSVD and TIK methods.

The filter factors in the TSVD method (also called pseudo-inverse filter) are defined to be one for large singular value, and zero for the rest of them [9]. More precisely, the filter factors for TSVD method are 
given by

$$
\phi_{i} \equiv \begin{cases}1 & i=1, \ldots, k \\ 0 & i=k+1, \ldots, N,\end{cases}
$$

where $k$ is the truncation parameter satisfying $1 \leq k \leq N$, which determines the number of the SVD components in the regularized solution (3.2).

The filter factors for the TIK method [9] are defined as

$$
\phi_{i} \equiv \frac{\sigma_{i}^{2}}{\sigma_{i}^{2}+\alpha^{2}}, \quad i=1, \ldots, N,
$$

where the parameter $\alpha>0$ is the regularization parameter. Tikhonov solution is related to the minimization problem

$$
\min _{x}\left\{\|b-A x\|_{2}^{2}+\alpha^{2}\|x\|_{2}^{2}\right\},
$$

which yields

$$
x_{f i l t}=\sum_{i=1}^{N} \frac{\sigma_{i}^{2}}{\sigma_{i}^{2}+\alpha^{2}} \frac{u_{i}^{T} b}{\sigma_{i}} v_{i} .
$$

The filter factors of the Tikhonov solution [9] satisfy

$$
\phi_{i}= \begin{cases}1-\left(\frac{\alpha}{\sigma_{i}}\right)^{2}+O\left(\left(\frac{\alpha}{\sigma_{i}}\right)^{4}\right), & \sigma_{i} \gg \alpha, \\ \left(\frac{\sigma_{i}}{\alpha}\right)^{2}+O\left(\left(\frac{\sigma_{i}}{\alpha}\right)^{4}\right), & \sigma_{i} \ll \alpha .\end{cases}
$$

The relation (3.7) comes from the Taylor expansion

$$
(1+\epsilon)^{-1}=1-\epsilon+\frac{1}{2} \epsilon^{2}+O\left(\epsilon^{3}\right)
$$

in the following relation:

$$
\phi_{i}= \begin{cases}\frac{1}{1+\alpha^{2} / \sigma_{i}^{2}}, & \sigma_{i} \gg \alpha, \\ \frac{1}{1+\sigma_{i}^{2} / \alpha^{2}}, & \sigma_{i} \ll \alpha .\end{cases}
$$

\subsection{Experimental results}

In this section we will apply the operator $E(Y)$, given by equation (2.3), on the image restoration process, in order to demonstrate the usefulness of the proposed method. Our basic idea is to use the output (3.2) as an appropriate choice of the matrix $Y$. The reconstruction defined by (3.2)-(3.3) is denoted by $Y=T S V D$ and the the reconstruction defined by (3.2)-(3.4) is denoted by $Y=$ TIK. On the other hand, numerical results corresponding to $E(Y=T S V D)$ and $E(Y=T I K)$ are presented and compared with the corresponding results generated by $Y=T S V D$ and $Y=T I K$. The experiments are performed using Matlab programming language [10] on an Intel(R) Core(TM)i3 CPU M380 @ 2.53 GHz 64 bit system with 2 GB of RAM memory running on the Windows 7 Enterprise.

The improvement in the quality of the original image $F\left(n_{1}, n_{2}\right)$ over the recorded blurred one $G\left(n_{1}, n_{2}\right)$ in the image restoration is measured by the signal-to-noise ratio $(S N R)$ improvement:

$$
I S N R=S N R_{\widetilde{F}}-S N R_{G}=10 \log _{10}\left(\frac{\sum_{n 1, n 2}\left(G\left(n_{1}, n_{2}\right)-F\left(n_{1}, n_{2}\right)\right)^{2}}{\sum_{n 1, n 2}\left(\widetilde{F}\left(n_{1}, n_{2}\right)-F\left(n_{1}, n_{2}\right)\right)^{2}}\right) .
$$


Note that all of the above signal-to-noise measures can only be computed in case when the ideal image is available, i.e., in an experimental setup or in a design phase of the restoration algorithm.

The peak signal-to-noise ratio (PSNR) is defined as the ratio between a signal's maximum power and the power of the signal's noise. Reasonably, a greater rate on PSNR is better since it shows that the ratio of SNR is higher. In this paper we use the following definition for PSNR:

$$
\text { PSNR }=20 \log _{10}\left(\frac{\max \left\{F\left(n_{1}, n_{2}\right)\right\}}{\sqrt{\left(1 /\left(n_{1} * n_{2}\right)\right) \sum_{i=0}^{n_{1}} \sum_{j=0}^{n_{2}}(F(i, j)-\widetilde{F}(i, j))^{2}}}\right),
$$

where $\max \left\{F\left(n_{1}, n_{2}\right)\right\}$ is the largest possible value of the original image and the denominator is defined as the root mean square difference between the original and the reconstructed images. The unit of both measures ISNR and PSNR is given in $d B$. We consider the blurred image that has been degraded by a uniform linear motion in the horizontal direction. A uniform linear motion blur is modeled by the matrix equation (2.2). The length of the blurring process is denoted by $l$, which implies $h_{i}=1 / l, i=-u, \ldots, u$.

Data corresponding to standard 8-bit grayscale test image 'Lena' are displayed on figures 3.1 and 3.2. The left (resp. right) graphic in Figure 3.1 displays data which are generated applying the Tikhonov (resp. $T S V D$ ) image deblurring method based on the FFT (Fast Fourier Transforms) algorithm in the computation of the spectral decomposition (3.1) of the matrix $A$.

The data obtained by TIK image deblurring method that uses the FFT algorithm and the TSVD image deblurring that uses the FFT algorithm are denoted by $T I K_{\mathrm{fft}}$ and $T S V D_{\mathrm{fft}}$, respectively. Figures 3.1 and 3.2 display data corresponding to zero boundary and reflexive boundary conditions, respectively.
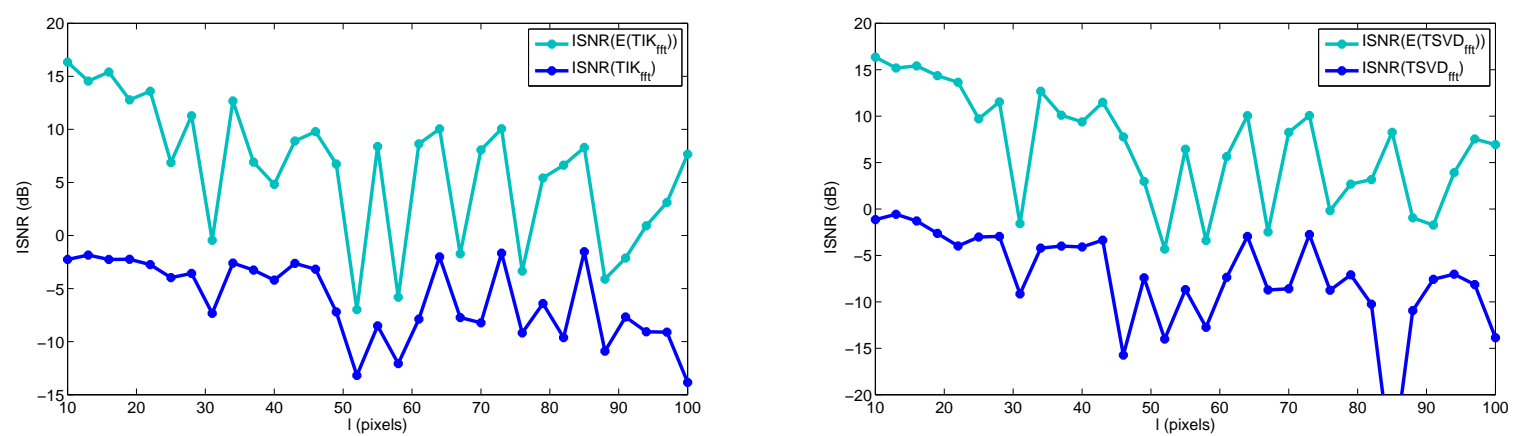

Figure 3.1. ISNR versus length $l$ for Lena and zero boundary conditions
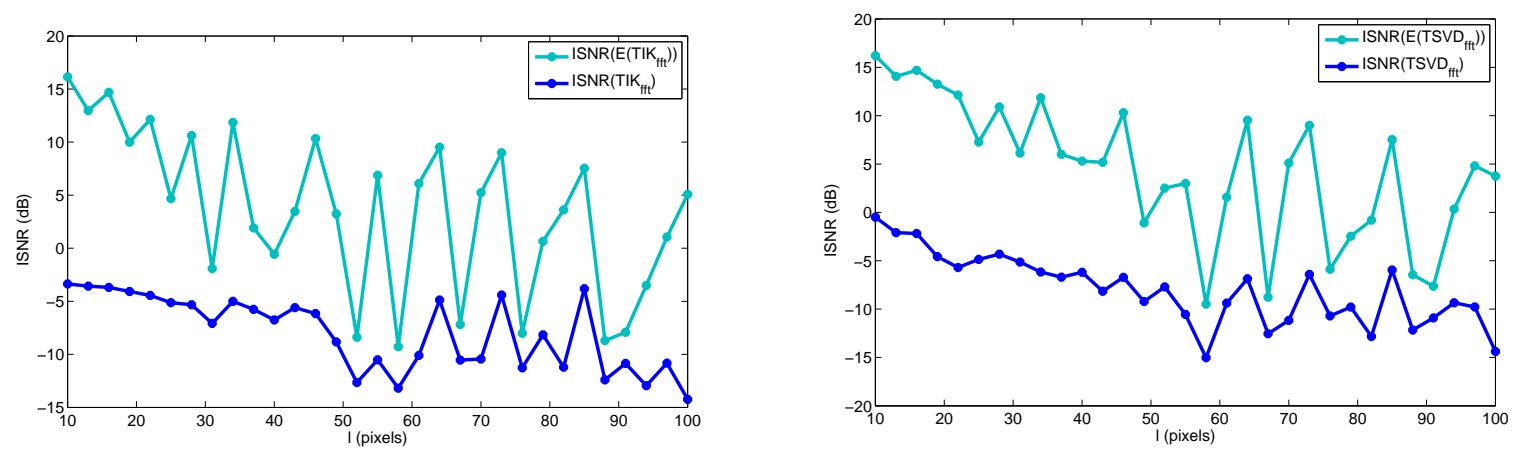

Figure 3.2. ISNR versus length $l$ for Lena and reflexive boundary conditions 
In addition, another standard image restoration example, the image "Barbara" will be used for the experiments presented in figures 3.3 and 3.4. The graphs placed in Figure 3.1 (resp. Figure 3.2) are similar to corresponding graphs illustrated in Figure 3.3 (resp. Figure 3.4).
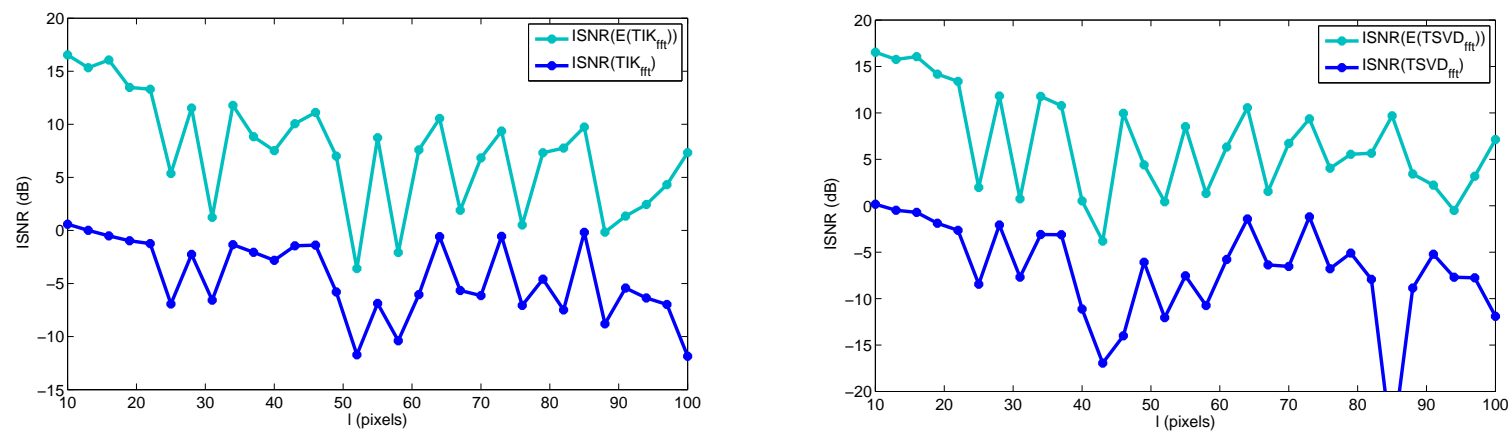

Figure 3.3. ISNR versus length $l$ for Barbara and zero boundary conditions
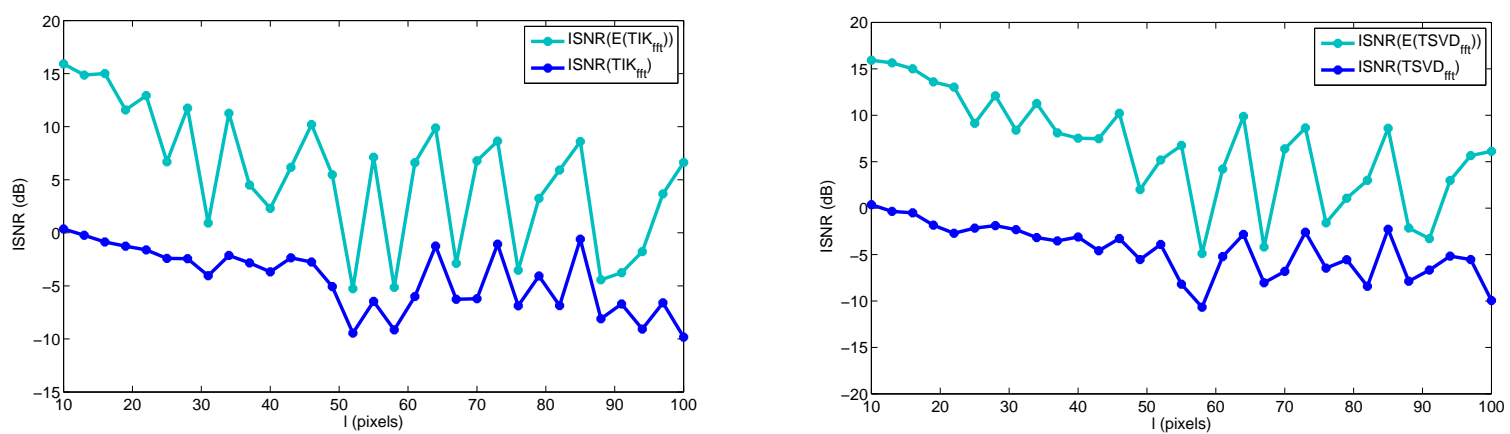

Figure 3.4. ISNR versus length $l$ for Barbara and reflexive boundary conditions

Figure 3.5 displays the PSNR values under the periodic boundary conditions in TIK and TSVD image deblurring methods which are based on the usage of Kronecker decomposition [9]. The data generated in this way are presented with the subscript SEP in Figure 3.5.
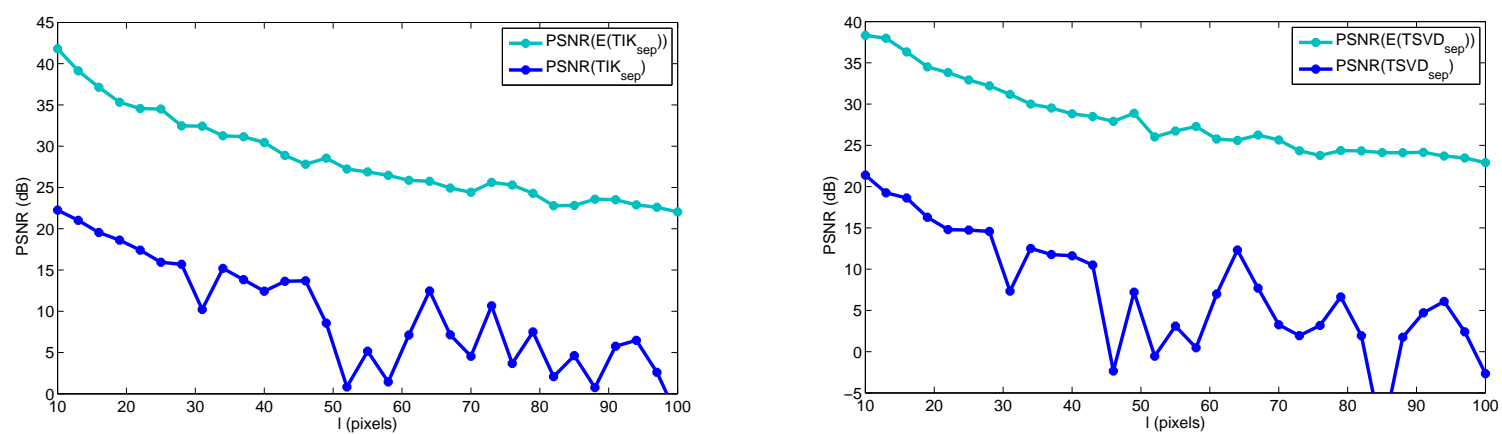

Figure 3.5. PSNR versus length $l$ of the uniform blurring process and periodic boundary conditions

Confirmation of the numerical experience that the image restorations $E(T I K)$ and $E(T S V D)$, obtained by the operator $E$ defined in (2.3), are better than the corresponding restoration produced by a direct application of the TIK and TSVD methods could be observed from figures 3.6, 3.7 and 3.8. 


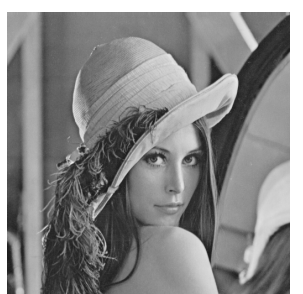

(a) Original image

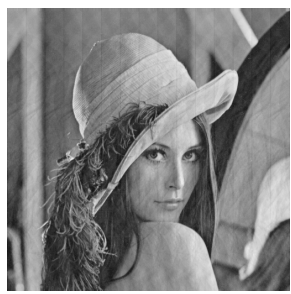

(d) E(TIK) restore

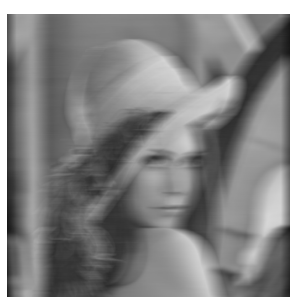

(b) Blurred image

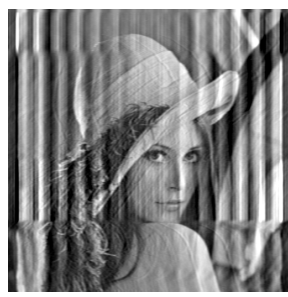

(e) TSVD restore

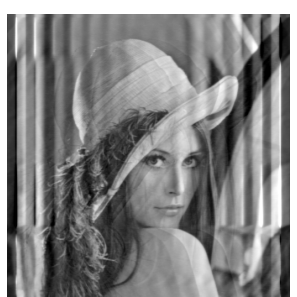

(c) TIK restore

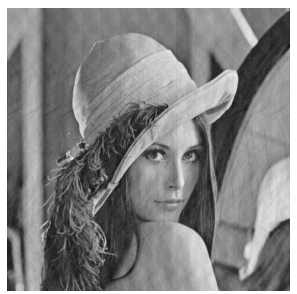

(f) E(TSVD)

Figure 3.6. Removal of blur length $l=34$ on a Lena image with zero boundary conditions.

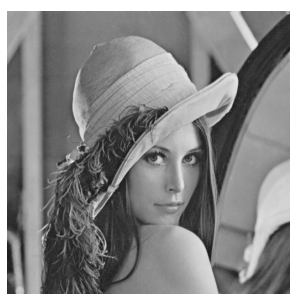

(a) Original image

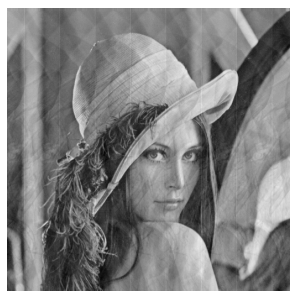

(d) $\mathrm{E}(\mathrm{TIK})$ restore

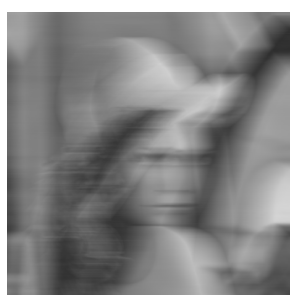

(b) Blurred image

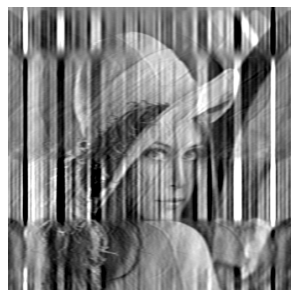

(e) TSVD restore

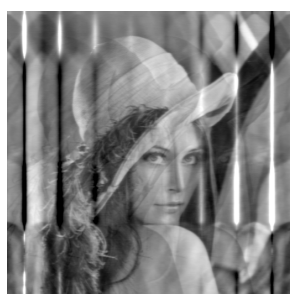

(c) TIK restore

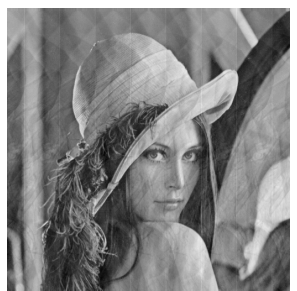

(f) E(TSVD)

Figure 3.7. Removal of blur length $l=64$ on a Lena image with reflexive boundary conditions.

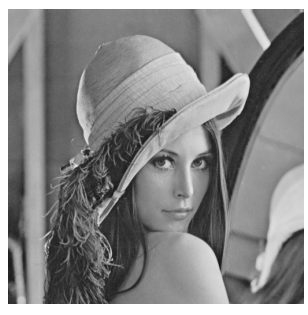

(a) Original image

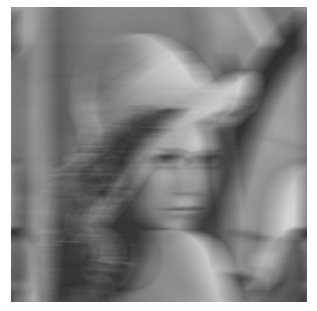

(b) Blurred image

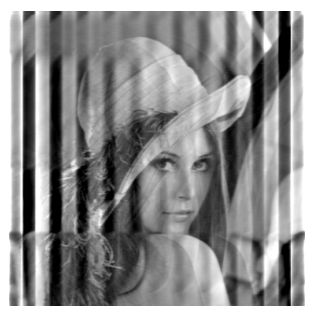

(c) TIK restore

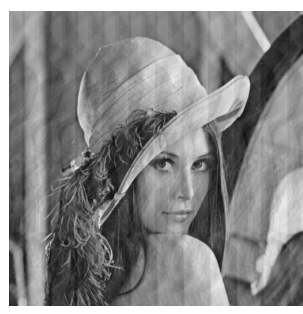

(d) E(TIK)

Figure 3.8. Removal of blur length $l=46$ on a Lena image with periodic boundary conditions. 


\section{Statistical Analysis of the Data Related with the Least Squares Restoration Method}

In this section we perform statistical analysis of the values $\operatorname{ISNR}(Y)$ and $\operatorname{ISNR}(E(Y))$ generated in this paper as well as in the papers $[19,20]$.

\subsection{Correlation and Regression}

First of all, linear regression was used in order to explore a possible relationship between the independent values $x=\operatorname{ISNR}(Y)$ and the dependent values $y=\operatorname{ISNR}(E(Y))$. The results are arranged in Table 4.1. The second column of Table 4.1 denotes the choices of restorations $Y$. The values in the third column denote the correlation coefficients of the linear regression established between $x$ and $y$. The expression of the linear regression is included in the fourth column. The following image restoration methods were applied on blurred images in order to obtain the restorations (given by the matrix $Y$ ) in Table 4.1:

(a) Haar 195 is the Haar based reconstructed image for $k=l=195$;

(b) Haar55 denotes the Haar based reconstructed image for $k=l=55$;

(c) Fourier 195 is the Fourier based reconstructed image for $k=l=195$;

(d) Fourier55 denotes the Fourier based reconstructed image for $k=l=55$;

(e) Constrained least-squares filter (CLS);

(f) Wiener filter (WF);

(g) Lucy-Richardson algorithm (LR).

These results are obtained from Section 3 of the present paper as well as from the published papers $[19,20]$.

It easy to observe that all regression lines included in Table 4.1 are increasing functions, which implies $\operatorname{ISNR}\left(Y\left(l_{1}\right)\right)>\operatorname{ISNR}\left(Y\left(l_{2}\right)\right) \Longrightarrow \operatorname{ISNR}\left(E\left(Y\left(l_{1}\right)\right)\right)>\operatorname{ISNR}\left(E\left(Y\left(l_{2}\right)\right)\right)$,

for two arbitrary blur lengths $l_{1}$ and $l_{2}$. Also, the values of correlation coefficients which are arranged in the second column of Table 4.1, always confirm the positive relationship greater than 0.7 between $x$ and $y$.

Additionally, we are looking for the interval $L$ which ensures $\operatorname{ISNR}(E(Y))>\operatorname{ISNR}(Y)$, for each value $\operatorname{ISNR}(Y)$ inside $L$. Those intervals are placed in the last column of Table 4.1 and they essentially mean intervals in which the operator $E$ may be used to improve $\operatorname{ISNR}(Y)$ values, for given $Y$. Any interval presented in the last column of Table 4.1 is defined by solving the inequality $y>x$, where $y=a * x+b$ denotes the corresponding regression line presented in the fourth column. For example, in the case $Y=$ Fourier 55 it is necessary to solve the inequality $y=0.7369 x+6.835>x$, which implies $x<25.969$.

Table 4.1. Correlation and linear regression between the ISNR values of $Y$ and $E(Y)$

\begin{tabular}{|c|c|c|c|c|}
\hline No & $Y$ & Correlation coefficient & Regression line & $L$ \\
\hline 1 & $Y=$ Fourier55 & 0.7094 & $y=0.7369 x+6.835$ & $(-\infty, 25.969)$ \\
\hline 2 & $Y=T I K_{f f t}$ & 0.7485 & $y=1.284 x+14.022$ & $(-49.373, \infty)$ \\
\hline 3 & $Y=$ Haar195 & 0.8602 & $y=1.9423 x-7.723$ & $(8.196, \infty)$ \\
\hline 4 & $Y=$ Haar55 & 0.9192 & $y=1.361 x+9.0685$ & $(-25.120, \infty)$ \\
\hline 5 & $Y=0$ & 0.9236 & $y=0.3040 x+7.9086$ & $(-\infty, 11.363)$ \\
\hline 6 & $Y=$ Fourier 195 & 0.9403 & $y=0.4432 x+15.526$ & $(-\infty, 27.884)$ \\
\hline 7 & $Y=L R$ & 0.9562 & $y=5.334 x-3.992$ & $(0.921, \infty)$ \\
\hline 8 & $Y=T I K_{D C T}$ & 0.9586 & $y=0.7494 x+40.553$ & $(-\infty, 161.824)$ \\
\hline 9 & $Y=C L S$ & 0.9750 & $y=2.267 x-5.855$ & $(4.621, \infty)$ \\
\hline 10 & $Y=W F$ & 0.9756 & $y=2.255 x-5.794$ & $(4.616, \infty)$ \\
\hline
\end{tabular}

\subsection{Other statistical measurements}

\subsubsection{Correlation quality and Average absolute difference}

Another numerical quantity that is presented in this section is the so-called correlation quality of an image. The indices produced by this measurement quantify the amount of distortion presented in an image. The original image Lena is tested against the blurred image and the images restored by applying different 
deblurring methods, as it is described in this work. The correlation quality $(C Q)$ is defined by

$$
C Q=\frac{1}{m s} \frac{\sum_{x=1}^{m} \sum_{y=1}^{s} I(x, y) \tilde{I}(x, y)}{\sum_{x=1}^{m} \sum_{y=1}^{s} I(x, y)},
$$

where $I$ denote the original image and $\tilde{I}$ is the reconstructed image. CQ retruns the correlation quality between the images $F$ and $\tilde{F}$. The CQ value is useful to quantitize the distortions on an image also in a watermarking process [16]. The size of the images is indicated by $m$ and $s$. The results corresponding to $C Q$ are presented in the third column of Table 4.2.

Finally, the values of the average absolute difference between the pixels of the original image and those produced by different image restoration methods is calculated. The average absolute difference between the pixels of the images $I$ and $I$ is another useful mesurement that quantitize the distortions on an image. It declares another numerical measurement that quantifies the amount of distortion presented in an image [16]. The average absolute difference $(A A V)$ is defined as the following double sum:

$$
A A V=\frac{1}{m s} \sum_{x=1}^{m} \sum_{y=1}^{s}[I(x, y)-\tilde{I}(x, y)]
$$

The results corresponding to $A A V$ are presented in the last column of Table 4.2. The values $C Q(Y)$ and $C Q(E(Y))$ as well as $A A V(Y)$ and $A A V(E(Y))$ are grouped in pairs.

It is clear that the reconstructed image is closer to the original one when the correlation quality is higher. We can observe from the values presented in Table 4.2 that all the restorations $E(Y)$ have better $C Q$ results than the corresponding methods $Y$. More precisely, the inequality $C Q(E(Y))>C Q(Y)$ holds for all considered image restorations $Y$.

It is also clear that smaller values of the Average Absolute difference between the pixels indicate that the reconstructed images are closer to their originals. We can see, from the values presented, that all restorations based on the usage of the operator $E(Y)$ have once more better values with respect to corresponding values of the restorations $Y$. More precisely, the inequality $A A V(E(Y))<A A V(Y)$ holds for all considered image restorations $Y$.

Table 4.2. Values for Correlation Quality and Average Absolute Difference

\begin{tabular}{l|l|c|c}
\hline \hline No & $Y$ & $C Q(Y)$ & $A A V(Y)$ \\
\hline \hline 1 & $Y=$ Blurred image for $n=34_{1}$ & $4.9028 \mathrm{e}-004$ & 10.8133 \\
\hline 2 & $Y=L R$ & $4.9392 \mathrm{e}-004$ & 9.9836 \\
3 & $Y=E($ LR $)$ & $4.9818 \mathrm{e}-004$ & 4.8959 \\
\hline 4 & $Y=$ Tik $_{f f t}$ & $4.9450 \mathrm{e}-004$ & 14.1211 \\
5 & $Y=E\left(\right.$ Tik $\left._{f f t}\right)$ & $4.9869 \mathrm{e}-004$ & 3.1300 \\
\hline 6 & $Y=T S D_{f f t}$ & $4.9507 \mathrm{e}-004$ & 18.7549 \\
7 & $Y=E\left(T S V D_{f f t}\right)$ & $4.9869 \mathrm{e}-004$ & 3.1313 \\
\hline 8 & $Y=C L S$ & $4.9766 \mathrm{e}-004$ & 4.6814 \\
9 & $Y=E(C L S)$ & $4.9847 \mathrm{e}-004$ & 3.3576 \\
\hline 10 & $Y=W F$ & $4.9766 \mathrm{e}-004$ & 4.6812 \\
11 & $Y=E($ WF $)$ & $4.9847 \mathrm{e}-004$ & 3.3576 \\
\hline 12 & $Y=O$ & $4.9789 \mathrm{e}-004$ & 5.6655 \\
\hline 13 & $Y=$ Haar $_{55}$ & $4.9872 \mathrm{e}-004$ & 2.9984 \\
14 & $Y=E\left(\right.$ Haar $\left._{55}\right)$ & $4.9877 \mathrm{e}-004$ & 2.9321 \\
\hline 15 & $Y=$ Fourier $_{55}$ & $4.9873 \mathrm{e}-004$ & 2.9535 \\
16 & $Y=E\left(\right.$ Fourier $\left._{55}\right)$ & $4.9878 \mathrm{e}-004$ & 2.9246 \\
\hline
\end{tabular}




\begin{tabular}{l|l|c|c}
\hline \hline No & $Y$ & $C Q(Y)$ & $A A V(Y)$ \\
\hline \hline 17 & $Y=$ Fourier $_{195}$ & $4.9874 \mathrm{e}-004$ & 2.9499 \\
18 & $Y=$ E $\left._{\text {(ourier }} 195\right)$ & $4.9881 \mathrm{e}-004$ & 2.8867 \\
\hline 19 & $Y=$ Haar $_{195}$ & $4.9875 \mathrm{e}-004$ & 2.9838 \\
20 & $Y=$ E(Haar $\left._{195}\right)$ & $4.9878 \mathrm{e}-004$ & 2.9246 \\
\hline \hline
\end{tabular}

Overall, the best results are obtained for the Fourier and Haar moment based reconstructed images. Although to a small extent, the Haar case is slightly better than the Fourier case. Obviously, increasing the number of coefficients, i.e. the number of moments, we succeed to have a recovered image that is closely related to the original image at a small time computational cost. In some cases, there would be no need to exceed a certain number of the coefficients. A small number of moments can retain important features of an image.

\subsubsection{Goodness of fit tests}

In this subsection we will examine whether the independent random datasets of values $\operatorname{ISNR}(Y)$ and $\operatorname{ISNR}(E(Y))$, for all different choices of the matrix $Y$, are drawn from the same underlying continuous population. In order to check this hypothesis on a 5\% significance level, we performed a KolmogorovSmirnov (K-S) test. We performed numerical experiments for all cases of the matrix $Y$ and the corresponding values of $E(Y)$.

Based on this test we find that $Y=0$ and $E(Y=0)$ are the only restorations where the ISNR values come from the same distribution.

In addition, we also find that the Wiener filter and the CLS are two restoration methods when both the ISNR and the PSNR values come from the same distribution. This conclusion can be verified from the values in Table 4.2, where the values for the images reconstructed by using these two methods are almost identical.

\section{Conclusions}

In this paper we finalize investigations from $[19,20]$. Specifically, we give further study about the possibilities of the application of the least squares solution $E(Y)$ in reconstructing blurred images. We firstly consider the possibility of improving the Tickhonov and TSVD image restoration methods. The main advantage of the proposed approach was found in the improvements of ISNR and PSNR. In this study, we present the results by comparing our method with the Tickhonov and TSVD method.

The second aim of our work is to perform a statistical analysis of the results obtained by applying the operator $E$, defined by equation (2.3), on previously generated restorations.

The final objective of this paper was the recovery of an image from degraded observations caused by linear motion. This approach can be applied in several scientific areas including medical imaging and diagnosis, military surveillance, satellite and astronomical imaging, and remote sensing.

\section{References}

[1] M. R. Banham, A. K. Katsaggelos, Digital image restoration, IEEE Signal Process. Mag. 14(2) (1997), $24-41$.

[2] J. Biemond, R. L. Lagendijk, R. M. Mersereau, Iterative methods for image deblurring, Proc. IEEE. 78(5) (1990), 856-883.

[3] A. Bovik, The essential guide to the image processing, Academic Press, 2009.

[4] S. Chountasis, V. N. Katsikis, D. Pappas, Applications of the Moore-Penrose inverse in digital image restoration, Mathematical Problems in Engineering, Volume 2009, Article ID 170724, 12 pages doi:10.1155/2009/170724.

[5] S. Chountasis, V. N. Katsikis, D. Pappas, Digital image reconstruction in the spectral domain utilizing the Moore-Penrose inverse, Mathematical Problems in Engineering, Volume 2010, Article ID 750352, 14 pages doi:10.1155/2010/750352.

[6] S. M. Chuiko, Least-squares method in the theory of ill-posed linear boundary-value problem with pulse action, Ukrainian Mathematical Journal 62 (2010) 690-697.

[7] Y. W. (Daniel) Fan, J.G. Nagy, Synthetic boundary conditions for image deblurring, Linear Algebra Appl. 434 (2011), $2244-2268$.

[8] R. C. Gonzalez and R. E. Woods, Digital Image Processing, 2nd Edition, Prentice-Hall, 2002. 
[9] P. C. Hansen, J. G. Nagy, D. P. O'Leary, Deblurring images: matrices, spectra, and filtering, SIAM, Philadelphia, 2006.

[10] Image Processing Toolbox User's Guide, The Math Works, Inc., Natick, MA, 2009.

[11] A. K. Katsaggelos, editor, Digital Image Restoration, Springer Verlag, New York, 1991.

[12] A. K. Katsaggelos, Iterative Image Restoration Algorithms, Digital Signal Processing Handbook, Ed. Vijay K. Madisetti and Douglas B. Williams Boca Raton: CRC Press LLC, 1999.

[13] D. Kundur, D. Hatzinakos, Blind image deconvolution: an algorithmic approach to practical image restoration, IEEE Signal Process. Mag. 13(3) (1996), 43-64.

[14] R. L. Lagendijk, J. Biemond, Iterative Identification and Restoration of Images, Kluwer Academic Publishers, Boston, MA, 1991.

[15] S. Miljković, M. Miladinović, P. S. Stanimirović, I. Stojanović, Application of the pseudoinverse computation in reconstruction of blurred images, Filomat 26 (2012), 453-465.

[16] F. A. P. Petitcolas, Watermarking schemes evaluation, I.E.E.E. Signal Processing 17(5) (2000), 58-64.

[17] I. V. Sergienko, Expansion of weighted pseudoinverse matrices with singular weights into matrix power products and iteration methods, Ukrainian Mathematical Journal 59 (2007) 1269-1289.

[18] Y. Shi, Q. Chang, Acceleration methods for image restoration problem with different boundary conditions, Appl. Numer. Math. 58 (2008), 602-614.

[19] P. S. Stanimirović, S. Chountasis, D. Pappas, I. Stojanović, Removal of blur in images based on least squares solutions, Mathematical Methods in the Applied Sciences 36 (2013), 2280-2296.

[20] P. S. Stanimirović, S. Chountasis, D. Pappas, I. Stojanović, Image deblurring process based on separable restoration methods, Comp. Appl. Math. 33 (2014), 301-323.

[21] P. S. Stanimirović, M. Miladinović, I. Stojanović, S. Miliković, Application of the partitioning method to specific Toeplitz matrices, Int. J. Appl. Math. Comput. Sci. 23 (2013), 809-821.

[22] X. Yu, Y. Gao, X.Yang, X. Yang, Image restoration method based on least-squares and regularization and fourth-order partial differential equations, Inform. Tech, J. 9 (2010), 962-967. 